\title{
Correction to: Examining the effects of teen dating violence prevention programs: a systematic review and meta-analysis
}

\section{Chelsey Lee $^{1} \cdot$ Jennifer S. Wong ${ }^{1}$}

Published online: 24 March 2021

(C) Springer Nature B.V. 2021

\section{Correction to: Journal of Experimental Criminology https://doi.org/10.1007/s11292-020-09442-x}

The original version of the article unfortunately contained mistakes. The table formatting of Tables 3 and 5 were incorrectly displayed in the PDF version of the article. The correct table formatting of these tables are given below.

The original article has been corrected.

Publisher's note Springer Nature remains neutral with regard to jurisdictional claims in published maps and institutional affiliations.

The online version of the original article can be found at https://doi.org/10.1007/s11292-020-09442-x

Jennifer S. Wong

jenwong@sfu.ca

Chelsey Lee

chelseyl@sfu.ca

1 School of Criminology, Simon Fraser University, 8888 University Drive, Burnaby, BC V5A 1S6, Canada 


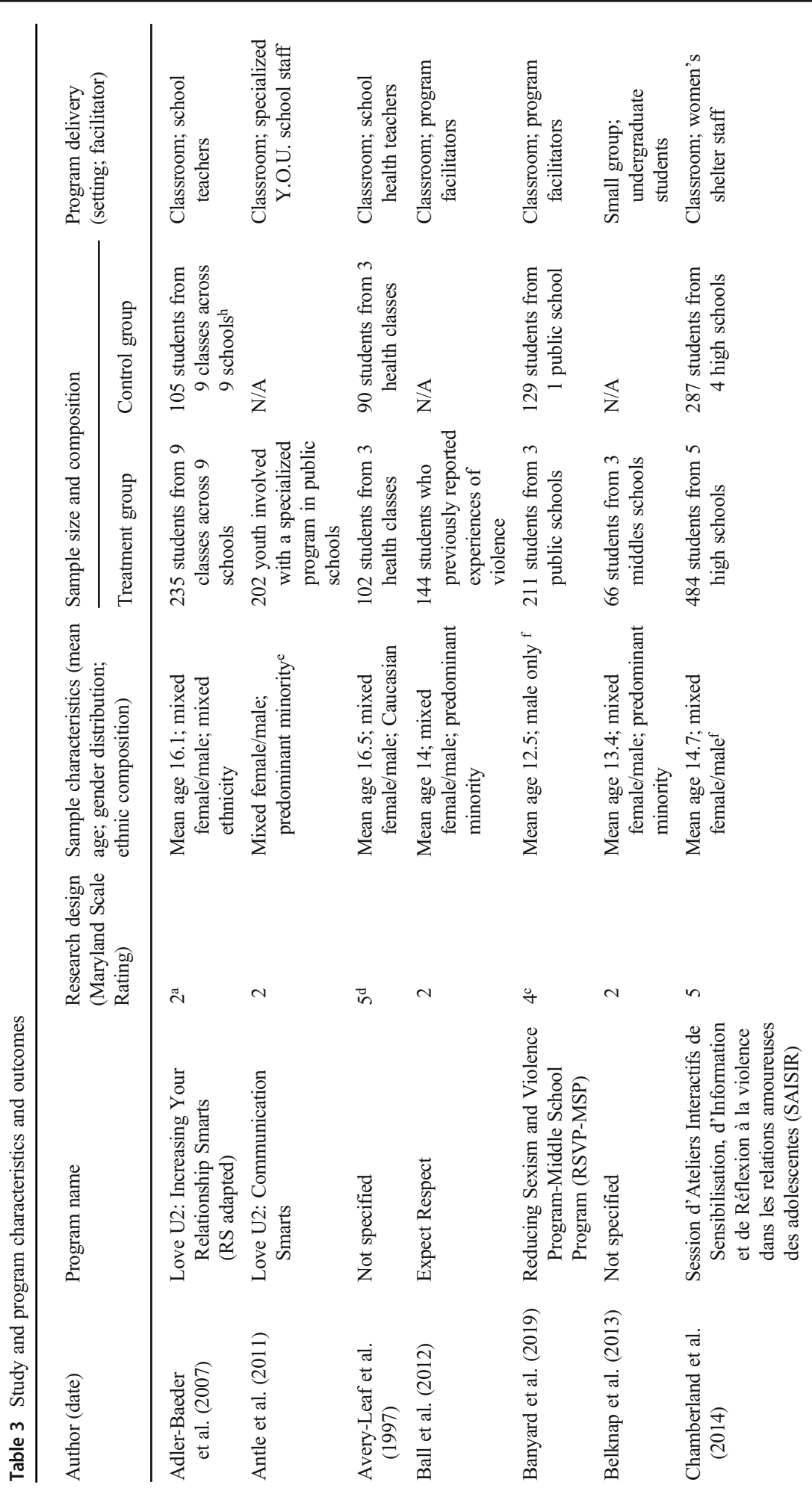




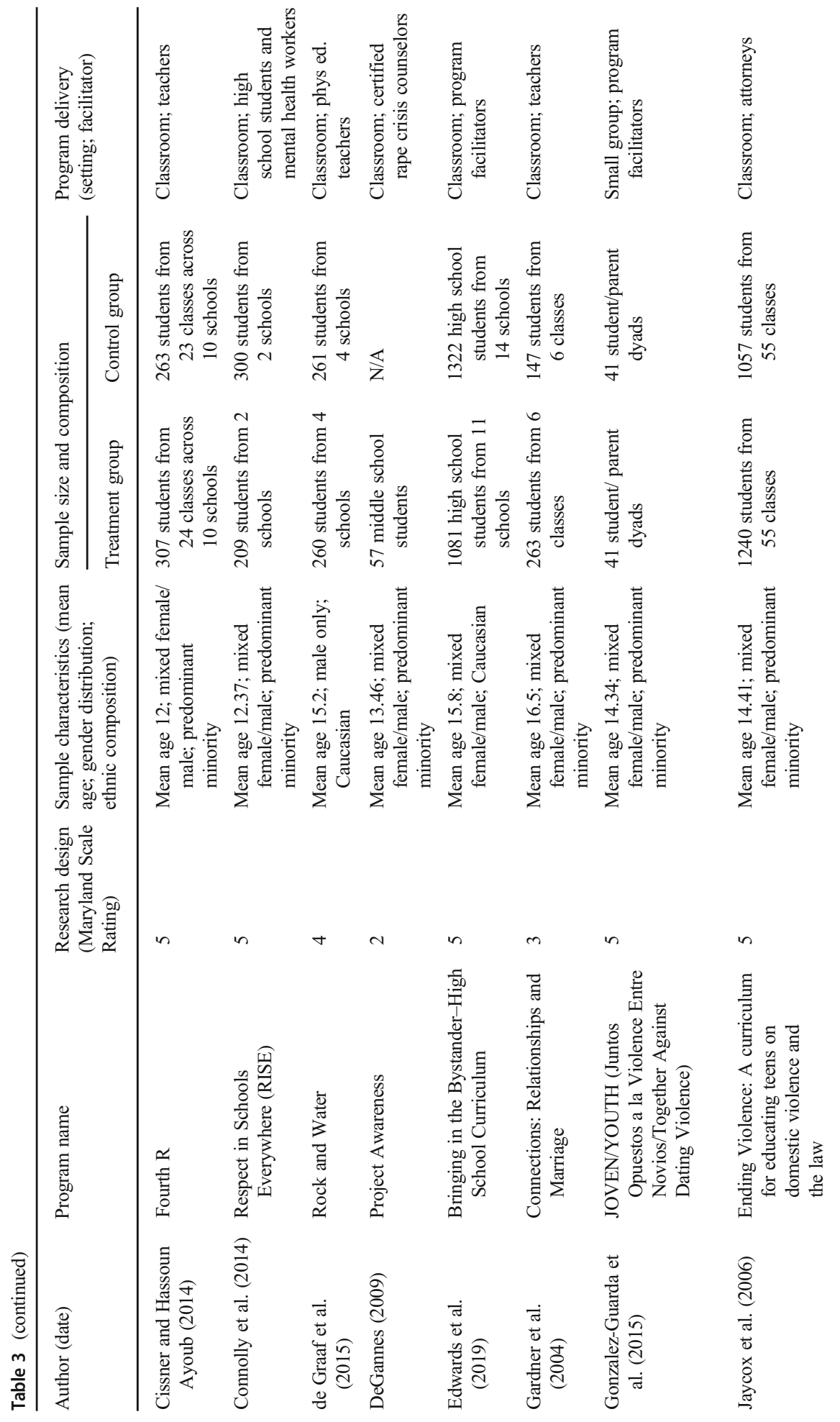




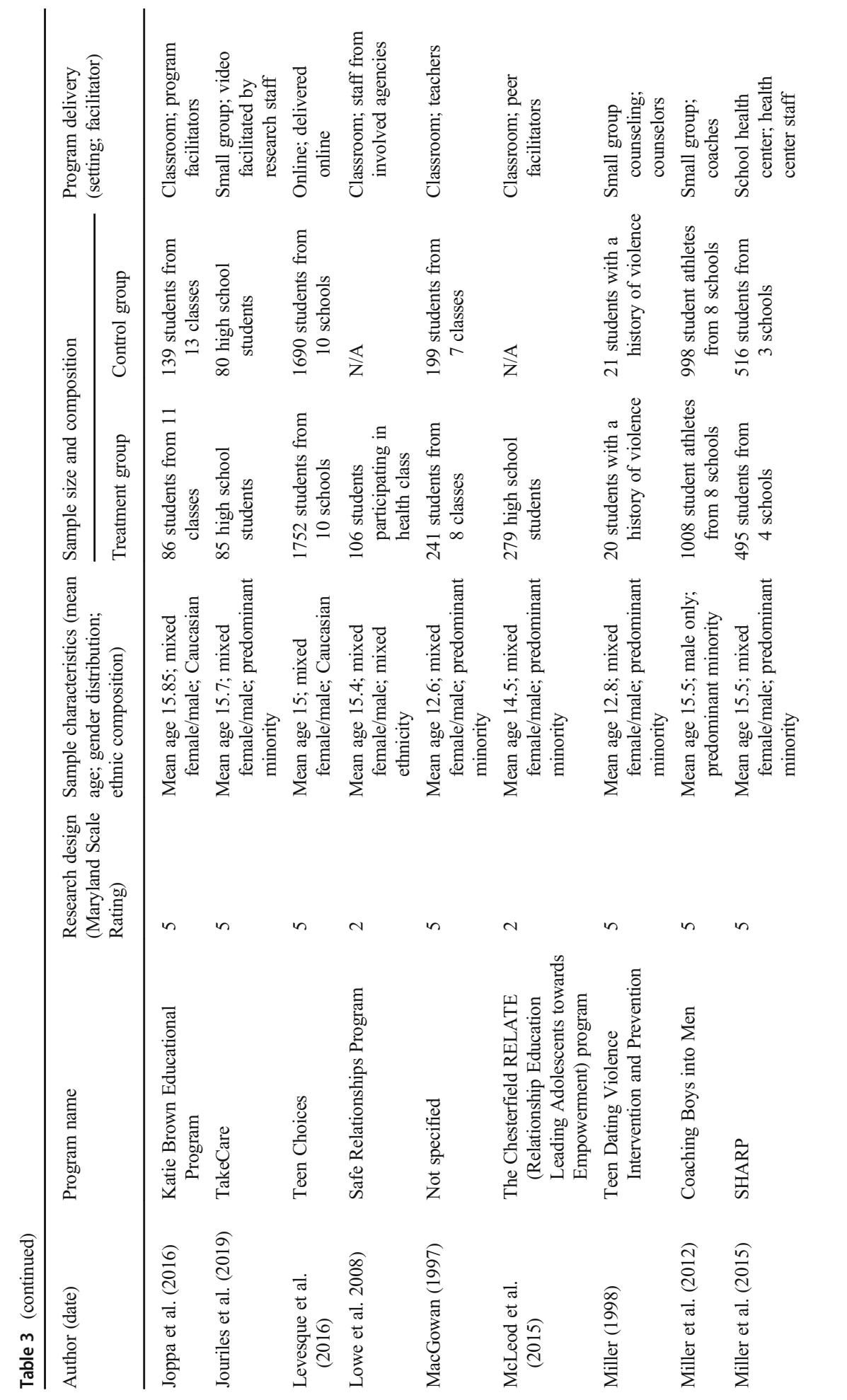




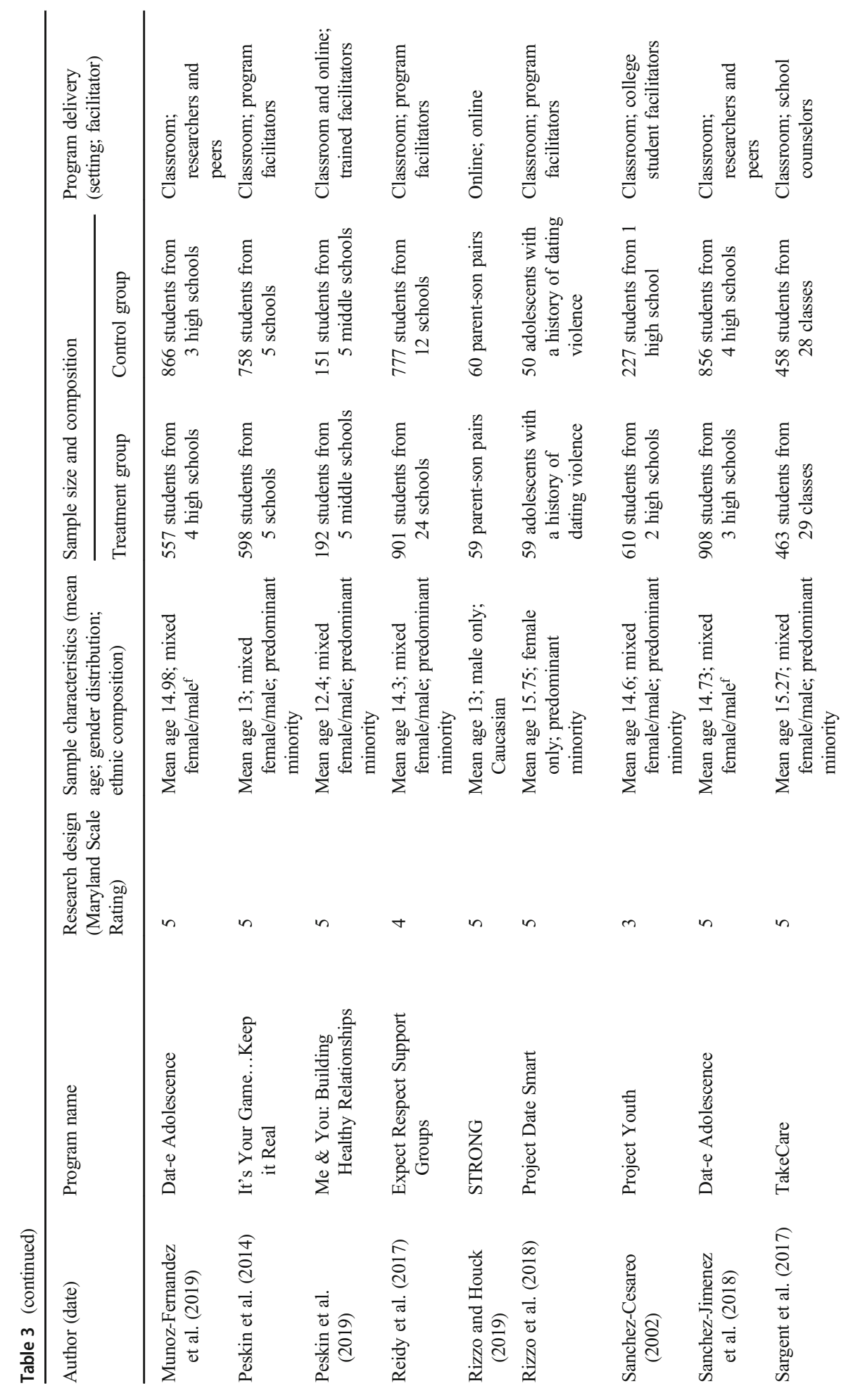




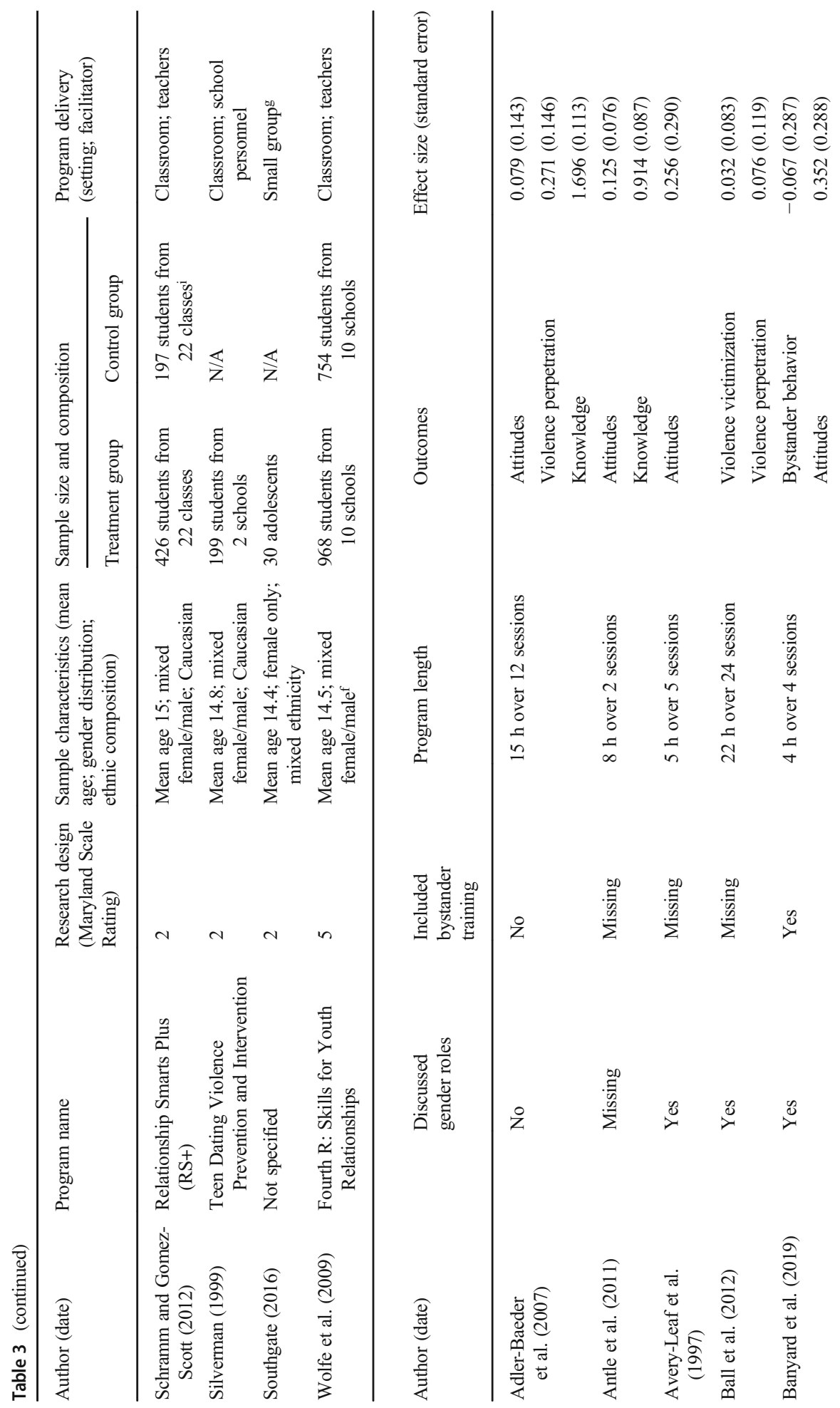




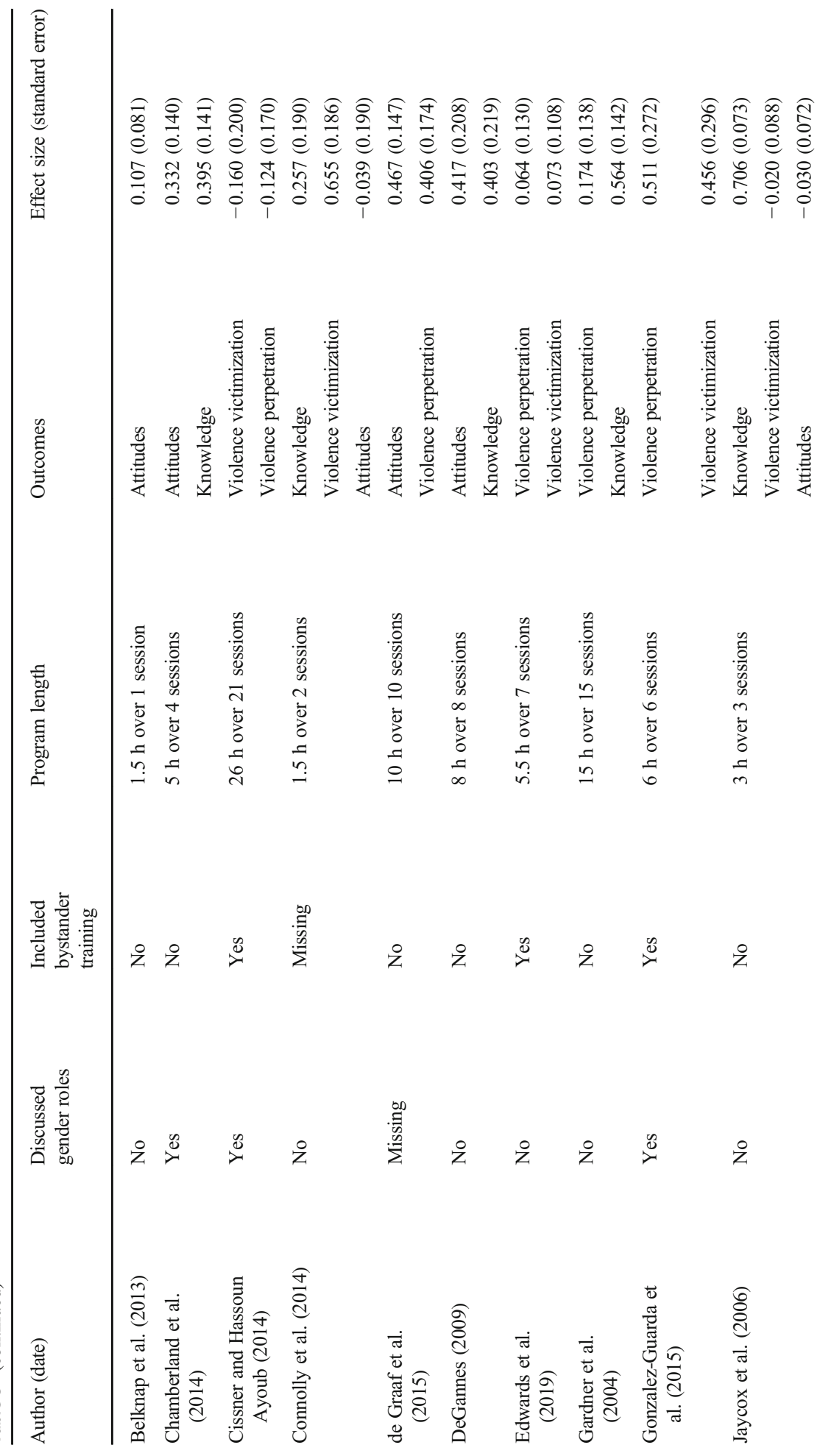




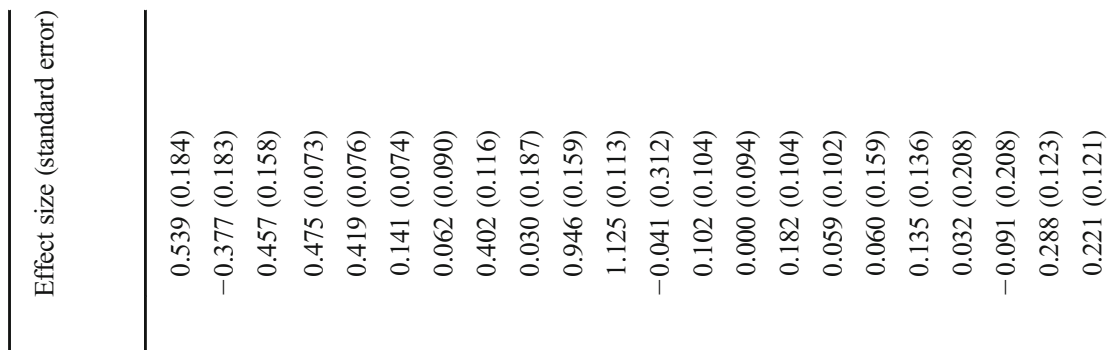

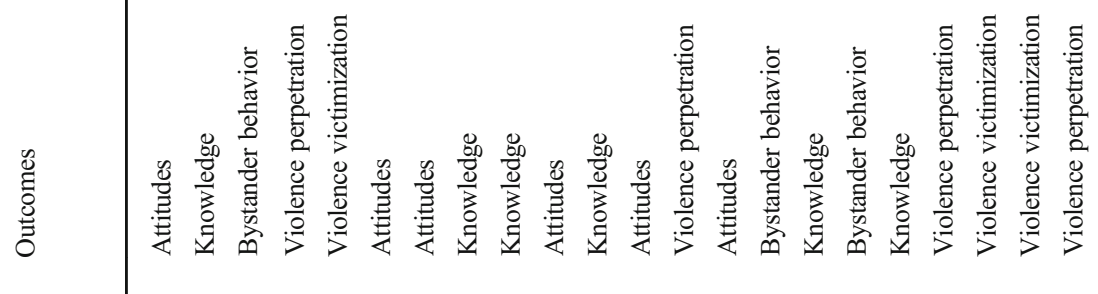

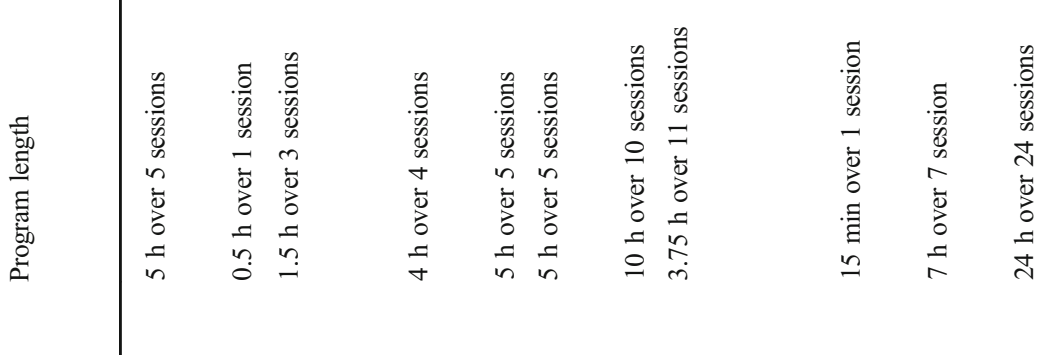

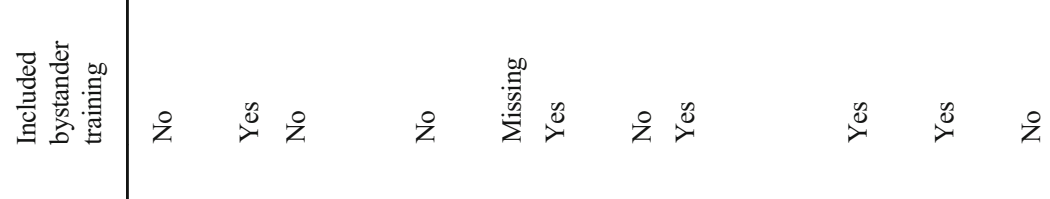

웡

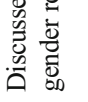

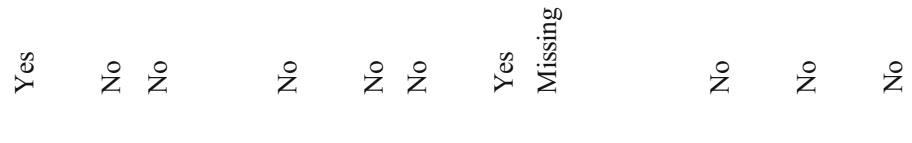

จิ 


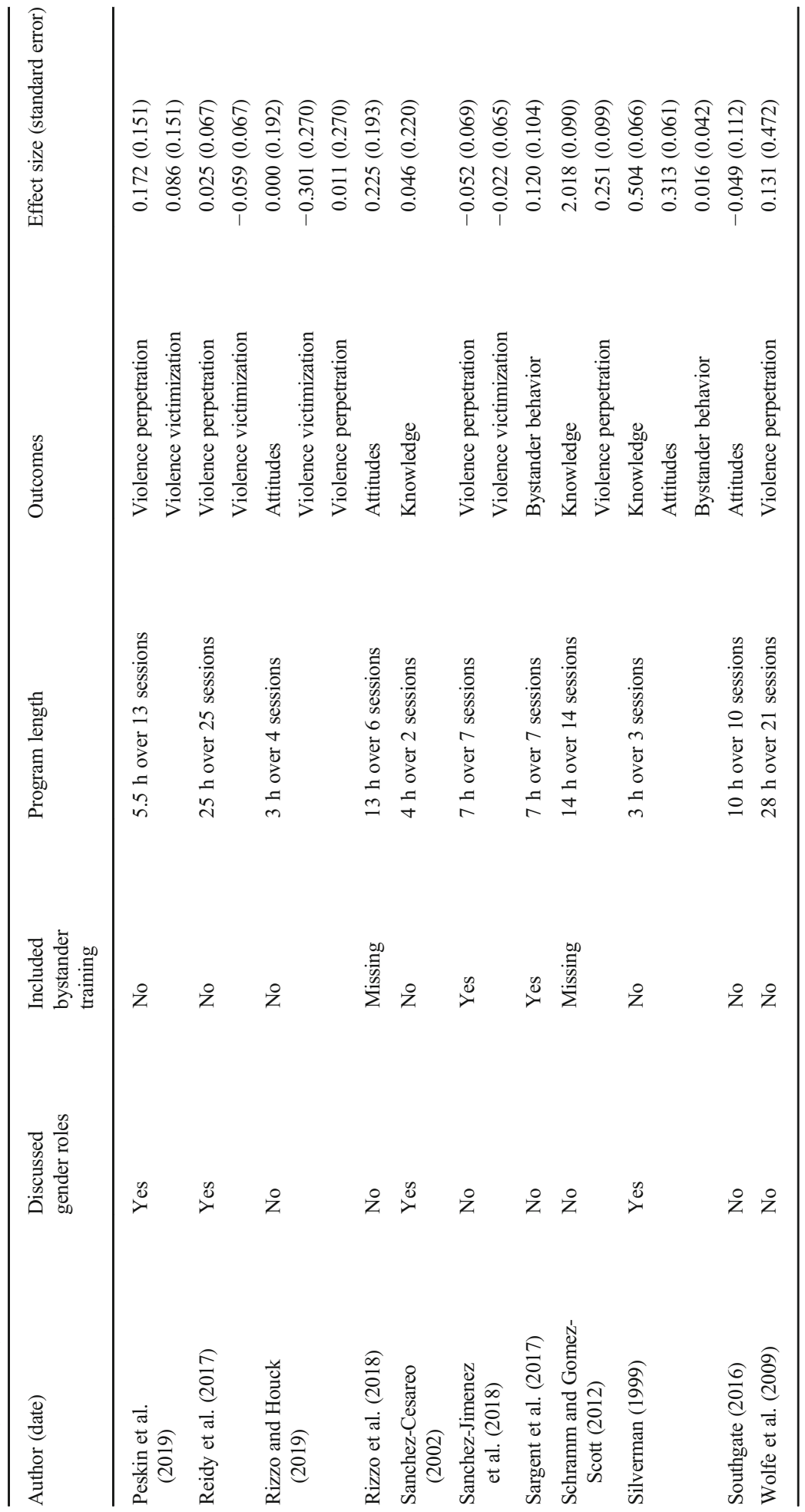


‡ कं

닐

急 焉

:

苞

帝 ह

言

盛

कू

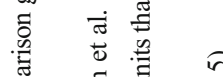

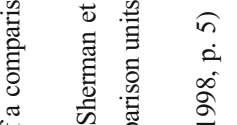

苂 है है जे

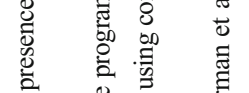

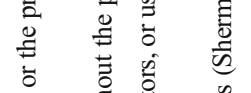

过苛 产

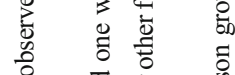

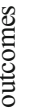

离离壱

气

蒙

音

s.

ส

च हूँ

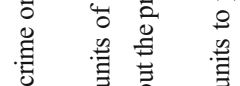

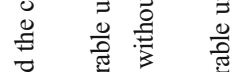

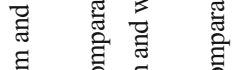

है

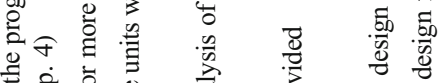

ల్

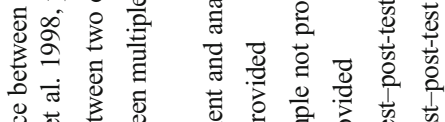

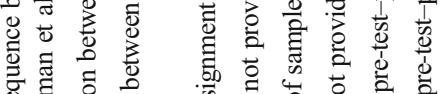

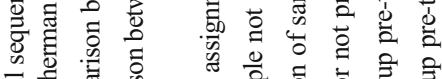

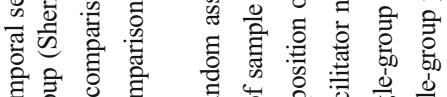

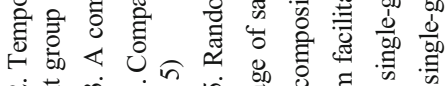

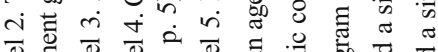

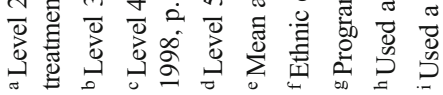

글 Springer 
Table 5 Summary of subgroup analyses by outcome

Outcome

\begin{tabular}{llll}
\hline Knowledge & Attitudes & Perpetration & Victimization
\end{tabular}

Program characteristics

Gender roles

$\begin{array}{lllll}\text { No } & E S=.946, z=25.4 * * * & E S=.104, z=3.2^{* *} & E S=.192, z=5.3^{* * *} & E S=.135, z=3.7 * * * \\ \text { Yes } & E S=.380, z=7.0^{* * *} & E S=.323, z=6.3^{* * *} & E S=.055, z=1.1 & E S=-.008, z=0.2 \\ & Q_{B}=122.2 * * * & Q_{B}=20.4^{* * *} & Q_{B}=7.2 * * & Q_{B}=5.8^{*}\end{array}$

Bystander training

No N/A

Yes

N/A

Program length

$\begin{array}{lll}<5 \mathrm{~h} & E S=.452, z=13.4^{* * *} & E S=.166, z=5.8^{* * *} \\ >5 \mathrm{~h} & E S=1.33, z=26.9 * * * & E S=.143, z=2.9^{* *} \\ & Q_{B}=215.7^{* * *} & Q_{B}=0.2\end{array}$

Setting

Middle school N/A

High school

Study characteristics

Sample size

Less than 300
300 or more
$E S=.694, z=19.0^{* * * *}$
$E S=.780, z=18.0^{* * * *}$
$Q_{B}=2.3$

Randomized design

$\begin{array}{ll}\text { No } & E S=.957, z=27.3^{* * * *} \\ \text { Yes } & E S=.342, z=7.5^{* * * *} \\ & Q_{B}=113.4 * * *\end{array}$

SGPP design

Non-SGPP

SGPP

$E S=.351, z=8.2 * * *$
$E S=1.01, z=27.5^{* * *}$
$Q_{B}=136.0 * * *$

Publication year

Prior to 2015

2015-2019

$$
\begin{aligned}
& E S=.774, z=25.6^{* * *} \\
& E S=.476, z=6.5 * * * \\
& Q_{B}=14.3^{* * *}
\end{aligned}
$$

$E S=.113, z=1.81$

$E S=.175, z=6.07 * * *$

$Q_{B}=1.0$

N/A
$E S=.228, z=5.9 * * * \quad E S=.115, z=3.0 * *$ $E S=.013, z=0.3$
$Q_{B}=13.6^{* * *}$
$E S=0.00, z=0.0$
$Q_{B}=13.2 * * *$

N/A

$E S=.245, z=4.6^{* * *}$ $E S=.013, z=0.4$

$Q_{B}=13.1 * * *$

$E S=.190, z=2.5^{*}$

$E S=.063, z=2.0 *$

$Q_{B}=2.4$
$E S=.205, z=6.8^{* * * *}$
$E S=.069, z=1.6$
$Q_{B}=6.7^{*}$

$E S=.207, z=3.1 *$
$E S=.137, z=4.4 * * *$
$Q_{B}=0.9$

$E S=.124, z=1.8$

$E S=.074, z=2.3 *$

$Q_{B}=0.4$
$E S=.209, z=6.5^{* * *}$
$Q_{B}=5.6^{*}$

$E S=.136, z=3.1 * *$

$E S=.159, z=4.4 * * *$

$Q_{B}=0.12$

N/A

$E S=.116, z=3.2 * * *$

N/A

N/A

$E S=.200, z=5.9^{* * *}$

$Q_{B}=2.92$

Time of post-test

Immediate
1 month+

$E S=.790, z=26.6^{* * *}$

$E S=.285, z=3.5^{* * * *}$

$Q_{B}=34.3 * * *$

$E S=.134, z=4.6^{* * *}$
$E S=.225, z=4.9^{* * *}$
$Q_{B}=2.8$

$E S=.156, z=3.4 * *$

$E S=.145, z=4.1 * *$

$Q_{B}=0.04$

$E S=.047, z=0.9$

$E S=.097, z=2.8 * *$

$Q_{B}=0.7$

$E S=.156, z=5.7 * * *$

$E S=.175, z=3.2 * *$

$Q_{B}=0.1$

$E S=.093, z=2.1 *$

$E S=.004, z=0.1$

$E S=.190, z=5.2 * * *$

$Q_{B}=2.9$

$E S=.142, z=3.7 * * *$

$Q_{B}=5.7^{*}$

Sample ethnicity

Caucasian/mixed

Predom minority

$E S=.963, z=22.4 * * *$

$E S=.574, z=15.2 * * *$

$Q_{B}=51.9 * * *$

$E S=.192, z=5.5^{* * *}$

$E S=.111, z=3.1 * *$

$E S=.328, z=6.9 * * *$

$E S=.091, z=2.2 *$

$Q_{B}=25.0 * * *$

$E S$ Effect size, $Q_{B} Q$-between group statistic, $N / A$ moderator not examined

$* p<.05, * * p<.01, * * * p<.001$ 